\title{
Estudio Morfométrico de la Región Orbitaria para la Instalación de Implantes Craneofaciales
}

\author{
Morphometric Study of Orbital Region for Craneofacial Implant Applications
}

\author{
"Sergio Olate; **Paulo Hemerson de Moraes; ${ }^{* * *}$ Paulo Henrique Ferreira Caria; \\ *****élgica Vásquez \& ** José Ricardo de Albergaria Barbosa
}

\begin{abstract}
OLATE, S.; DE MORAES, P. H.; CARIA, F. P. H.; VÁSQUEZ, B. \& BARBOSA, A. J. R. Estudio morfométrico de la región orbitaria para la instalación de implantes craneofaciales. Int. J. Morphol., 29(1):22-26, 2011.

RESUMEN: El objetivo de esta investigación es establecer las relaciones morfométricas presentes en las regiones periorbitarias utilizadas para la instalación de implantes craneofaciales. Se diseñó un estudio descriptivo utilizando 40 cráneos humanos de entre 20 y 60 años del Laboratorio de Anatomía del Departamento de Morfología de la Facultad de Odontología de Piracicaba de la Universidad Estadual de Campinas. Fueron realizadas tomografías volumétricas cone beam y a partir de la reconstrucción virtual se precisaron los puntos de análisis, basados en la literatura científica, tanto clínica como anatómica de la región. Se realizaron divisiones de la órbita respetando distancias mínimas y reparos anatómicos como el seno frontal y seno maxilar. En las mediciones realizadas, se observó que el área supraorbitaria presentaba una distancia ósea sagital de $8,14 \mathrm{~mm} \pm 1,91 \mathrm{~mm}$, el reborde infraorbitario de $7 \mathrm{~mm} \pm 1,71 \mathrm{~mm}$ y el área lateral de órbita un promedio de 7,91 $\mathrm{mm} \pm 1,15 \mathrm{~mm}$. Considerando que los implantes de mayor dimensión son de $6 \mathrm{~mm}$, la instalación de implantes en estas regiones está totalmente asegurada en términos de requerimientos de cantidad ósea regional.
\end{abstract}

PALABRAS CLAVE: Implante craneofacial; Morfometría órbita; Rehabilitación facial.

\section{INTRODUCCIÓN}

La primera investigación realizada para estudiar las técnicas asociadas a la instalación de implantes craneofaciales o fijaciones extraorales, destinadas a la rehabilitación protésica maxilofacial, fue realizada por Tjellström et al. (1981) y consistió en la instalación de implantes en el área periauricular para aplicar una epítesis de oreja externa. A partir de esta experiencia, las técnicas de rehabilitación facial sobre implantes fueron indicadas a diferentes situaciones, como reconstrucciones posteriores a resecciones oncológicas, tratamiento de secuelas de trauma, tratamiento de malformaciones y deformidades faciales, entre otras (Tjellström et al., 1985, Wolfaardt et al., 2003).

La región orbitaria ha sido estudiada por algunos autores, señalando la previsibilidad del tratamiento (Jacobsson et al. 1992, Wolfaardt et al.); sin embargo, son limitados los estudios que relatan las características morfológicas de la región orbitaria, lo que limita la aplicación de la técnica y la conformación de protocolos de trata- miento. De hecho, existen sólo dos publicaciones que relacionan la morfometría facial y la instalación de implantes craneofaciales (Jensen et al., 1992, Matsuura et al., 2002); la metodología aplicada en ambos estudios fue similar, realizando mediciones con pie de metro en el trabajo de Jensen et al. y segmentaciones de los cráneos estudiados (Matsuura et al.).

En la región orbitaria, son dos las aplicaciones generales: epítesis de bulbo ocular y epítesis de bulbo ocularórbita; con ambas situaciones, la instalación de implantes craneofaciales está asociada, prácticamente, a cualquier reparo óseo existente (Wright et al., 2008); no obstante, la órbita, compuesta por siete huesos diferentes, presenta un seno frontal, un seno maxilar y células etmoidales en áreas próximas, de forma que es necesario identificar cuáles son las regiones exactas de intervención y sus características morfométricas para evitar invadir áreas nobles y aumentar la previsibilidad de los tratamientos.

** División de Cirugía Oral y Maxilofacial, Departamento de Odontología Integral, Facultad de Medicina, Universidad de La Frontera, Chile.

** Área de Cirugía Oral y Maxilofacial, Departamento de Diagnóstico Oral, Facultad de Odontología de Piracicaba, Universidad Estadual de Campinas, Brasil.

*** Área de Anatomía, Departamento de Morfología, Facultad de Odontología de Piracicaba, Universidad Estadual de Campinas, Brasil.

***** Facultad de Ciencias de la Salud, Universidad Autónoma de Chile, Chile. 


\section{MATERIAL Y MÉTODO}

Selección de la muestra y estudio de imagen. Fueron seleccionados 40 cráneos humanos, de entre 20 y 60 años de edad del Laboratorio de Anatomía del Departamento de Morfología de la Facultad de Odontología de Piracicaba de la Universidad Estadual de Campinas. Cada cráneo fue identificado con un número arbitrario y fue realizada una captura de imagen mediante tomografía volumétrica cone beam utilizando el sistema ICAT VISION. Con estas capturas fueron realizadas las reconstrucciones craneanas para estudiar la morfometría de la región orbitaria (Fig. 1).

Diseño del estudio y puntos de análisis. Las reconstrucciones fueron incluidas en el software InVesallius 3.0 para obtener las mediciones en los planos sagitales, coronales y axiales. La elección de los puntos de análisis fueron establecidos en la reconstrucción tridimensional según las investigaciones morfométricas de Jensen $e t$ al. y Matsuura et al. y las investigaciones clínicas de Wolfaardt et al. y Tjellström et al. Los puntos seleccionados fueron desde las areas externas de las corticales laterales y mediales en los diferentes cortes (Fig. 2).

La órbita fue dividida arbitrariamente en 9 sectores, asociados a las ubicaciones frecuentemente utilizadas en la práctica clínica. El punto de medición fue distante $5 \mathrm{~mm}$ del límite de la estructura ósea, respetando el grosor mínimo de instalación de implantes craneofaciales.

Los sectores son: órbita superior: parte del reborde supraorbitario lateral $(5 \mathrm{~mm})$ al seno frontal hasta el límite lateral de la órbita; las mediciones fueron realizadas cada $7 \mathrm{~mm}$ de distancia, estableciendo una distancia total aproximada de $20 \mathrm{~mm}$.
Órbita inferior: parte de los huesos maxilar y cigomático que conforman el reborde infraorbitario. Este sector fue dividido arbitrariamente en 4 regiones de igual distancia medio lateral realizando las mediciones en cada lugar de división (3 mediciones).

Órbita lateral: parte de los huesos frontal y cigomático que conforman la pared lateral de la órbita. Este sector fue dividido en dos áreas superior e inferior, siendo las mediciones realizadas en el punto medio de la mitad superior y en el punto medio de la mitad inferior (Fig. 2).

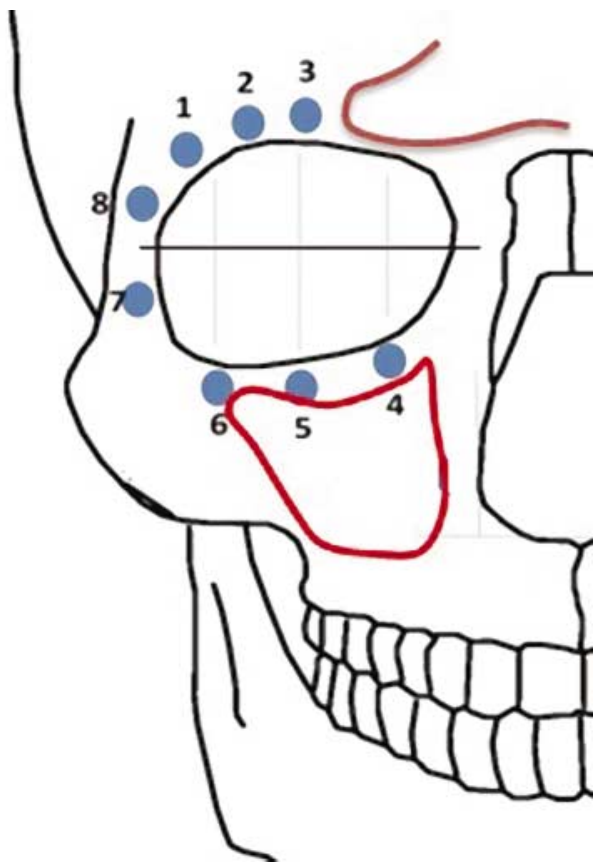

Fig. 2. Diseño de la órbita señalando los puntos utilizados que fueron seleccionados en base a la literatura científica. Se respetaron las distancias mínimas entreimplantes y desde el seno frontal.

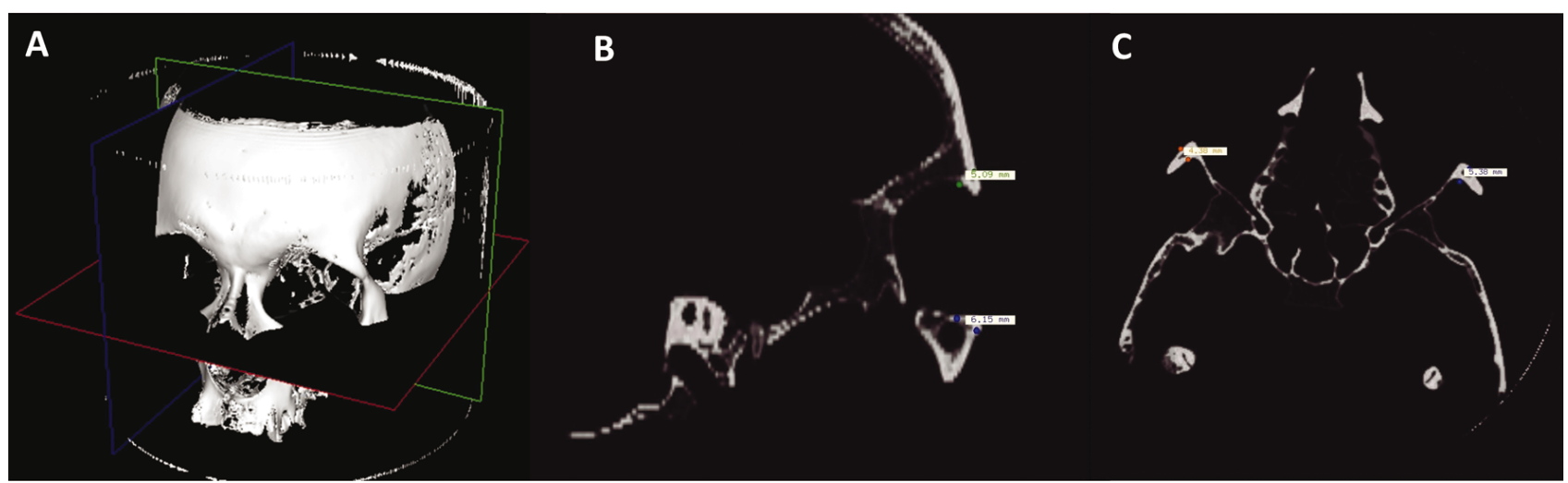

Fig.1. A) Reconstrucción volumétrica del cráneo mediante tomografía cone beam, donde se establecieron los puntos de medición. B) Imagen sagital donde se realizaron las mediciones anteroposteriores de órbita superior e inferior, en los lugares escogidos previamente. C) Imagen axial donde se realizaron mediciones anteroposteriores de la región lateral de órbita en los puntos seleccionados en la imagen volumétrica. 
Finalmente, fue realizado un estudio descriptivo de los hallazgos, siendo comparados con los resultados obtenidos por Jensen et al. y Matsuura et al.

\section{RESULTADOS}

En la región superior se observó una distancia aproximada de $23,5 \mathrm{~mm} \pm 6,63 \mathrm{~mm}$ (desde lateral del seno frontal). En la región superior fue observada una distancia anteroposterior promedio de $8,14 \mathrm{~mm} \pm 1,91 \mathrm{~mm}$. Las dimensiones individuales se presentan en la Tabla I.

En el reborde infraorbitario se observó una distancia anteroposterior promedio de $7 \mathrm{~mm} \pm 1,71 \mathrm{~mm}$. Los resultados individuales se presentan en la Tabla II.

La región lateral de órbita presentó una distancia anteroposterior promedio de $7,91 \mathrm{~mm} \pm 1,15 \mathrm{~mm}$. Las mediciones individuales sson presentadas en la Tabla III.

Tabla I. Dimensiones óseas anteroposteriores en la región del reborde supra orbitario en 40 cráneos humanos obtenidas mediante análisis con tomografía volumétrica cone beam.

\begin{tabular}{lcccccc}
\hline Órbita superior $(\boldsymbol{O S})$ & \multicolumn{2}{c}{ Lateral $(\mathrm{mm})$} & \multicolumn{2}{c}{ Mediana $(\mathrm{mm})$} & \multicolumn{2}{c}{ Medial $(\mathrm{mm})$} \\
\cline { 2 - 7 } & $X$ & $\begin{array}{c}\text { DE o } \\
\text { límites }\end{array}$ & $X$ & $\begin{array}{c}\text { DE o } \\
\text { límites }\end{array}$ & $\begin{array}{c}\text { DE o } \\
\text { límites }\end{array}$ \\
\hline Jensen et al. $(1992)$ & 4,6 & $3,0-6,9$ & - & - & 4,40 & $2,7-7,0$ \\
Matsuura et al. $(2002)$ & 9,9 & 3,3 & 16,0 & 3,1 & 9,50 & 1,90 \\
Grupo masc ulino & 7,73 & 1,86 & 8,15 & 1,81 & 9,05 & 2,55 \\
Grupo femenino & 7,14 & 1,33 & 8,53 & 1,8 & 9,52 & 2,18 \\
Total presente estudio & 7,5 & 1,69 & 8,31 & 1,8 & 9,23 & 2,40 \\
\hline
\end{tabular}

X: promedio; DE: desviación estándar; mm: milímetros.

Tabla II Dimensiones óseas anteroposteriores en la región del reborde infra orbitario en 40 cráneos humanos obtenidas mediante análisis con tomografía volumétrica cone beam.

\begin{tabular}{lcccccc}
\hline Órbita inferior $(\boldsymbol{O I})$ & \multicolumn{2}{c}{ Lateral $(\mathrm{mm})$} & \multicolumn{2}{c}{ Mediana $(\mathrm{mm})$} & \multicolumn{2}{c}{ Medial $(\mathrm{mm})$} \\
\cline { 2 - 7 } & $X$ & $\begin{array}{c}\text { DE o } \\
\text { límites }\end{array}$ & $X$ & $\begin{array}{c}\text { DE o } \\
\text { límites }\end{array}$ & $X$ & $\begin{array}{c}\text { DE o } \\
\text { límites }\end{array}$ \\
\hline Jensen et al. $(1992)$ & 5,4 & $2,6-7,1$ & - & - & - & - \\
Matsuura et al. $(2002)$ & 11,7 & 3,7 & 6,0 & 2,6 & 4,3 & 2,0 \\
Grupo masc ulino & 7,94 & 1,5 & 6,18 & 1,74 & 6,6 & 2,4 \\
Grupo femenino & 7,14 & 1,91 & 6,97 & 2,29 & 5,45 & 2,3 \\
Total presente estudio & 7,62 & 1,73 & 6,51 & 2,0 & 6,11 & 2,42 \\
\hline
\end{tabular}

X: promedio; DE: desviación estándar; mm: milímetros.

Tabla III. Dimensiones óseas anteroposteriores en la región del reborde lateral de órbita en 40 cráneos humanos obtenidas mediante análisis con tomografía volumétrica cone beam.

\begin{tabular}{lcccc}
\hline Orbita lateral $(\boldsymbol{O L})$ & \multicolumn{3}{c}{ Superior $(\mathrm{mm})$} & Inferior $(\mathrm{mm})$ \\
\cline { 2 - 5 } & $X$ & DE o límites & $X$ & DE o límites \\
\hline Jensen et al. $(1992)$ & 5,9 & $5,6-8,0$ & 6,1 & $4,0-7,2$ \\
Matsuura et al. $(2002)$ & 9,2 & 1,4 & 10,0 & 2,6 \\
Grupo masc ulino & 8,03 & 1,44 & 7,20 & 1,32 \\
Grupo femenino & 5,93 & 1,3 & 5,73 & 1,4 \\
Total presente estudio & 7,25 & 1,72 & 6,65 & 1,52 \\
\hline
\end{tabular}

X: promedio; DE: desviación estándar; mm: milímetros. 


\section{DISCUSIÓN}

La región orbitaria es muy importante en la rehabilitación facial. En ella debemos diferenciar situaciones asociadas a la pérdida del bulbo ocular o a la pérdida de estructuras óseas orbitaria. En el primer caso, la situación clínica puede abordarse con la instalación de implantes en cualquier estructura ósea adyacente, mientras que en el segundo caso, la instalación de implantes debe ser estratégicamente planificada debido a que el soporte óseo y la distribución de los implantes influyen en la rehabilitación protésica (Palmer et al. 2001, Tolman \& Desjardins 1991).

El seno frontal es una de las grandes limitaciones en el reborde supraorbitario y las investigaciones de Jensen $e t$ $a l$. y Matsuura et al. no abordan este tema; Jensen $e t$ al. establecen una distancia de $4,4 \mathrm{~mm}$ y Matsuura et al. de $3,0 \mathrm{~mm}$ a $5,0 \mathrm{~mm}$. Algunos autores señalan que a partir de los 19 años el seno frontal presenta una tendencia a aumentar su tamaño a causa de la pneumatización, lo que hace cada vez más complejo los procedimientos quirúrgicos a este nivel (Fatu et al., 2006). En nuestra muestra la distancia entre el límite lateral del seno frontal y el límite lateral del reborde supraorbitario fue de $23 \mathrm{~mm} \pm 5,74 \mathrm{~mm}$, siendo éste el lugar destinado a la implantación; desde lateral, el grosor óseo fue de $7,94 \mathrm{~mm}, 7,92 \mathrm{~mm}$ y 8,58 , lo cual permite la instalación de implantes sin problemas.

El contacto de implantes craneofaciales con el seno frontal no ha sido evaluado científicamente, pero podemos especular en base a los estudios asociados al seno maxilar, que en casos de invasiones menores de $2 \mathrm{~mm}$ podría existir recubrimiento epitelial total (Jeong et al. 2009); si consideramos que un implante craneofacial presenta entre $3 \mathrm{~mm}$ y $6 \mathrm{~mm}$, la invasión de $2 \mathrm{~mm}$ al seno deja aproximadamente $1 /$ 3 o más del implante sin contacto óseo, lo cual puede limitar la sobrevida del mismo. Por otra parte, en la región lateral de la órbita existe la posibilidad de instalar implantes sin complicaciones anatómicas relevantes; de hecho, esta área es frecuentemente abordada para el manejo del trauma facial con éxito en los tratamientos (Olate et al. 2010).

En el caso del reborde infraorbitario se evidenció una cantidad ósea adecuada, siendo ellos de 7,89mm, 6,52mm y $6,59 \mathrm{~mm}$ (desde lateral a medial); para Matsuura et al. las variaciones se presentaron entre $11,7 \mathrm{~mm}$ y $4,3 \mathrm{~mm}$ lo cual puede estar asociado a los diferentes puntos de medición utilizados por los autores. Esta región, a pesar de tener buenos resultados, es poco utilizada en la práctica clínica puesto que los lugares habituales de implantación para prótesis ocular están en la región superior y lateral; además, en casos complejos tratados con maxilectomías, esta región se encuentra ausente e inviable como punto de implantación (Palmer et al., Cervelli et al. 2006).

\section{CONCLUSIÓN}

Con los resultados de esta investigación, podemos señalar que es posible instalar con seguridad implantes craneofaciales en las regiones superior, lateral e inferior de órbita, respetando las distancias anatómicas de los senos frontal y maxilar. En las regiones estudiadas la cantidad ósea disponible es adecuada para instalar implantes de $6 \mathrm{~mm}$.

OLATE, S.; DE MORAES, P. H.; CARIA, F. P. H.; VÁSQUEZ, B. \& BARBOSA, A. J. R. Morphometric study of orbital region for craneofacial implant applications. Int. J. Morphol., 29(1):22-26, 2011.

SUMMARY: The aim of this research was to study the morphometric relation present in periorbital region, used in the installation of craniofacial implants. A descriptive study was carried out using 40 skulls between 20 and 60 years of age from the Laboratório de Anatomia do Departamento de Morfologia da Facultade de Odontologia de Piracicaba da Universidade Estadual de Campinas. Volumetric tomography with cone beam technique was used along with virtual reconstruction, with the point of analysis being selected based on the clinical and anatomical scientific literature. Orbital division was realized with minimal distance from the frontal and maxillary sinus. During measurement it was observed that the superior orbital rim presented a sagittal bone distance of $8.14 \mathrm{~mm} \pm 1.91 \mathrm{~mm}$; inferior orbital rim of $7 \mathrm{~mm} \pm 1.71 \mathrm{~mm}$, and the lateral orbital presented an average of $7.91 \mathrm{~mm} \pm 1.15 \mathrm{~mm}$, considering that craniofacial implants present up to $6 \mathrm{~mm}$ in length, the installation of implants in this area is considered safe in terms of regional bone quantity requirements.

KEY WORD: Craniofacial implants; Orbital morphometry; Facial rehabilitation.

\section{REFERENCIAS BIBLIOGRÁFICAS}

Cervelli, V.; Bottini, D., J.; Arpino, A.; Colicchia, G., M.; Mugnaini, F.; Trimarco, A.; Gentile, P. \& Grimaldi, M.
Orbital reconstruction: bone-anchored implants. $J$. Craniofac. Surg., 17:848-53, 2006. 
OLATE, S.; DE MORAES, P. H.; CARIA, F. P. H.; VÁSQUEZ, B. \& BARBOSA, A. J. R. Estudio morfométrico de la región orbitaria para la instalación de implantes craneofaciales. Int. J. Morphol., 29(1):22-26, 2011.

Fatu, C.; Puisoru, M.; Rotaru, M. \& Truta, A. M. Morphometric evaluation of the frontal sinus in relation to age. Ann. Anat., 188:275-80, 2006.

Jacobsson, M.; Tjellström, A.; Fine, L. \& Andersson, H. A retrospective study of osseintegrated skin-penetrating titanium fixtures used for retaining facial prostheses. Int. J. Oral Maxillofac. Implants, 7:523-8, 1992.

Jensen, O., T.; Brownd, C. \& Blacker, J. Nasofacial prostheses supported by osseointegrated implants. Int. J. Oral Maxillofac. Implants, 7:203-11, 1992.

Jeong, S.; Choi, B., H. \& Xuan, F. A retrospective study of the effects of sinus membrane elevation on bone formation around implants placed in the maxillary sinus cavity. Oral Surg. Oral Med. Oral Pathol. Oral Radiol. Endod., 107:364-8, 2009.

Matsuura, M.; Ohno, K.; Michi, K. \& Egawa, K. Clinicoanatomic study on the craniofacial bones used for cranio- and maxillofacial implants. Int. J. Oral Maxillofac. Implants, 17:121-9, 2002.

Olate, S.; Lima Jr, S., M.; Sawazaki, R., Moreira, R., W., F. \& Moraes, M. Surgical approaches and fixation patterns in zygomatic complex fractures. J. Craniofac. Surg., 21:1213-7, 2010.

Palmer, S.; Brix, M. \& Bénateau. Prothèse faciale complexe: intérêt des prostheses maxilla-faciales à ancrage osseoux dans le traitement des pertes de substance étendues de la face. Ver. Stomatol. Chir. Maxillofac., 102:261-5, 2001.

Tjellström, A.; Yontchev, E.; Lindström, J. \& Brånemark, P-I. Five years' experience with bone-anchored auricular prosthesis. Otolaryngol. Head Neck Surg., 93:366$72,1985$.

Tjellström, A.; Lindström, J.; Nylén, O.; Albrektsson, T.; Brånemark, P.-I.; Birgersson, B.; Nero, H. \& Sylvén, C. The bone-anchored auricular episthesis. Laryngoscope, 91:811-5, 1981.

Tolman, D., E. \& Desjardins, R., P. Extraoral application of osseointegrated implants. J. Oral Maxillofac. Surg., 49:33-45, 1991.

Wolfaardt, J.; Gehl, G.; Farmand, M. \& Wilkes, I. Indications and methods of care for aspects of extraoral osseointegration. Int. J. Oral Maxillofac. Surg., 32:12431, 2003.
Wright, R., F.; Zemnick, C., Wazen, J., J. \& Asher, E. Osseointegrated implants and auricular defects: a case series study. J. Prosthodontics, 17:468-75, 2008.

Dirección de correspondencia

Prof. Dr. Sergio Olate Morales

Departamento de Odontología Integral

División de Cirugía Oral \& Maxilofacial

Facultad de Medicina

Universidad de La Frontera

Claro Solar 115

Temuco

CHILE

Email:solate@ufro.cl

Recibido : 22-09-2010

Aceptado: 12-12-2010 